\title{
Luxación posterior bilateral de hombro
}

\section{Bilateral posterior shoulder dislocation}

\author{
Luis Justino Fernández Palomo, ${ }^{*}$ Ramón González Pola, ${ }^{*}$ Alejandro Miravete Gálvez*
}

\begin{abstract}
RESUMEN
La luxación posterior bilateral del hombro es una lesión extremadamente rara; se ha sugerido que es patognomónica de eventos convulsivos cuando se diagnostica en la ausencia de trauma. Casi $50 \%$ de las luxaciones posteriores bilaterales se debe a un evento convulsivo, lo cual aumenta a $90 \%$ si las luxaciones están asociadas con alguna otra fractura. Otra causa de esta patología es una descarga eléctrica, pero es causa de menos de $5 \%$ de las luxaciones posteriores bilaterales del hombro. La sospecha clínica es indispensable para el diagnóstico y el abordaje radiológico confirma el diagnóstico. En el presente escrito, se presentan dos casos de luxación posterior bilateral del hombro secundarias a eventos convulsivos, así como una revisión de la literatura.
\end{abstract}

Palabras clave: Luxación posterior, luxación de hombro, convulsión, electrocución.

Nivel de evidencia: IV

\section{INTRODUCCIÓN}

El hombro es la articulación que con mayor frecuencia presenta luxaciones, en la mayoría de los casos, anteriores. La luxación posterior de hombro es una patología poco frecuente que corresponde de 2 a $5 \%$ de las luxaciones de hombro, aunque de éstas, menos de $10 \%$

\footnotetext{
* Cirujano Ortopedista, Centro de Ortopedia y Traumatología.
}

Centro Médico ABC. Ciudad de México.

Recibido para publicación: 02/03/2020. Aceptado: 03/04/2020.

Correspondencia: Dr. Luis Justino Fernández Palomo Centro Médico ABC Santa Fe.

Av. Carlos Graef Fernández Núm. 154, Consultorio 522, Col. Tlaxala, 05300, Alcaldía Cuajimalpa de Morelos, CDMX.

Tel: (55) 1664 7075/7076

E-mail: ljfernandez@abchospital.com

\begin{abstract}
Bilateral posterior shoulder dislocation is an extremely rare injury, and it has been suggested that it is pathognomonic for seizure events when diagnosed in the absence of trauma. Almost $50 \%$ of bilateral posterior dislocations are due to a seizure event, this increases to $90 \%$ if the dislocations are associated with any other fracture. Although electric shock may also be the cause of this pathology, it represents less than $5 \%$ of bilateral posterior shoulder dislocations. A systematization of the clinical and radiological approach is essential, followed by an early diagnosis and appropriate surgical treatment. Two cases of bilateral posterior shoulder dislocation are presented, caused by a seizure event, as well as a review of the literature.
\end{abstract}

Keywords: Posterior fracture, dislocation, shoulder. Seizures, electrocution.

Level of evidence: $I V$

son bilaterales. ${ }^{1}$ La fractura o luxación bilateral es extremadamente rara, pues sólo se han reportado 35 casos en la literatura. La fractura más común causada por una convulsión es la del cuello quirúrgico del húmero. ${ }^{2}$ La fractura o luxación posterior del hombro es aun menos común, pues representa $0.9 \%$ de las 1,500 luxaciones y de las luxaciones revisadas por Neer. ${ }^{3,4}$

Se atribuyen diferentes etiologías a esta condición particular, como el «síndrome triple $\mathrm{E}$ (epilepsia o cualquier evento convulsivo, trauma extremo y descarga eléctrica)»; este «síndrome» representa las tres principales causas de una luxación bilateral posterior del hombro. ${ }^{2}$ Casi $50 \%$ de las luxaciones posteriores bilaterales suele ser causado por un evento convulsivo; este porcentaje aumenta a $90 \%$ si las luxaciones están asociadas con fracturas. ${ }^{5}$ La descarga eléctrica representa menos de 5\% de las luxaciones posteriores bilaterales del hombro y es mucho más común como causa de luxaciones posteriores unilaterales. ${ }^{2}$ 
Las convulsiones asociadas con la luxación de hombro pueden causar diferentes tipos de fracturas, pero la fractura del cuello y/o cabeza humeral es la más común, aunque no la única; se pueden asociar también con fracturas de escápula, fracturas en la columna vertebral, de acetábulo, cabeza femoral, fémur o pelvis. ${ }^{2}$ Respecto al hombro, el espasmo del músculo infraespinoso y redondo menor favorece una fractura del cuello del húmero, ya que se genera una contracción masiva no balanceada de los músculos de la cintura escapular en aducción, flexión y rotación interna; esto conlleva además a un choque de la cabeza humeral contra el acromion y la glenoides, permitiendo la luxación posterior y un bloqueo en el borde glenoideo, y creando una fractura por compresión en la cabeza humeral con una lesión de tipo Hill Sachs reversa.

\section{CASOS CLÍNICOS}

Se presentan dos casos de luxación posterior bilateral del hombro causados por eventos convulsivos, diagnosticados y tratados en el Centro Médico ABC Santa Fe por el autor principal.

\section{Caso 1}

Se presenta el caso de una mujer de 71 años, quien acudió al Servicio de Urgencias en periodo postictal de una hora de evolución, el cual es secundario a secuelas de evento vascular cerebral antiguo, que fue ya tratado; refiere dolor intenso en ambos hombros e imposibilidad para movilizarlos.

Se identificó la limitación funcional de ambas extremidades torácicas con actitud en rotación interna y aducción. No se identificó el compromiso neurovascular en ninguna de las extremidades; se realizaron estudios de radiología simple en dos proyecciones de ambos hombros, así como tomografía axial computada de cráneo y hombros (Figuras 1 y 2). Se identificó luxación glenohumeral posterior de ambos hombros con fractura de ambas cabezas femorales impactadas en el borde glenoideo.

La paciente fue llevada a sala de operaciones ese mismo día y se le realizó reducción abierta de ambas
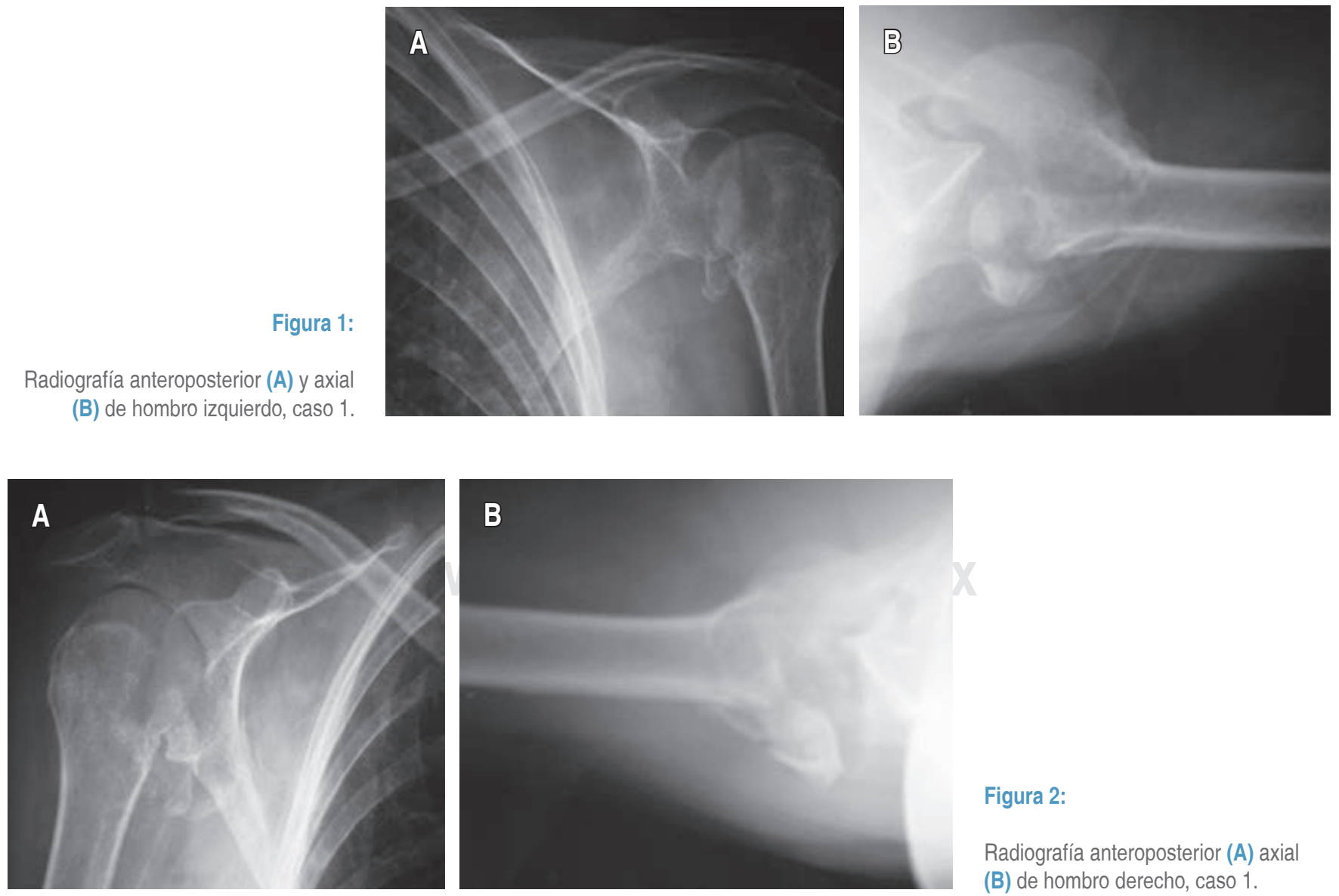

Figura 2:

Radiografía anteroposterior (A) axial (B) de hombro derecho, caso 1. 

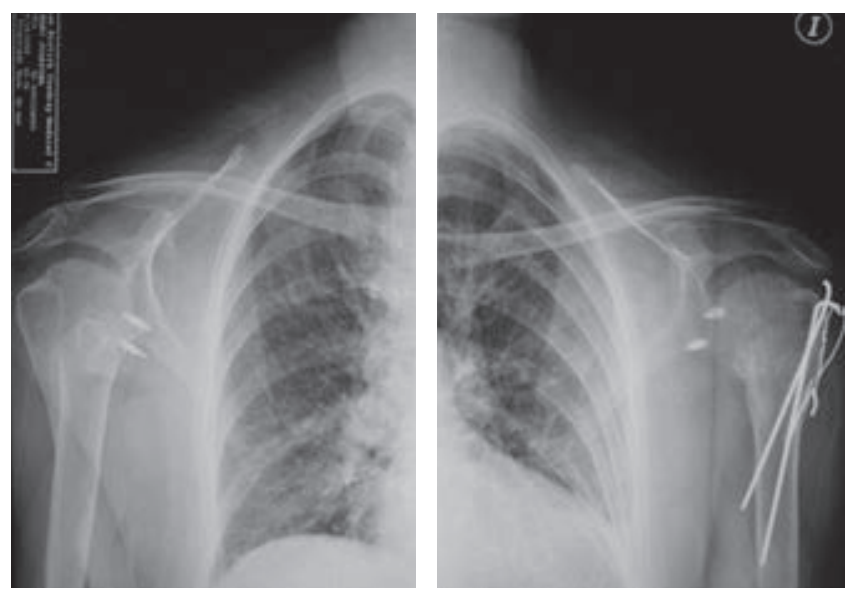

Figura 3: Radiografía anteroposterior, un año después de la cirugía de ambos hombros.

lesiones. En posición de silla de playa, en una mesa de hombro, se le realizó abordaje posterior transversal, siguiendo el trayecto infraespinoso e iniciando con el hombro derecho. Se identificó la lesión capsular con desprendimiento del labrum y la fractura de la cabeza femoral, así como desplazamiento de la tuberosidad mayor. Se realizó la reducción de la articulación, con colocación de injerto óseo y matriz en la zona del defecto de la cabeza y se realizó cerclaje con alambre y dos clavos de Kirshner; después se efectuó plastia capsular con dos anclas y reinserción del ligamento glenohumeral posterior con el labrum. Posteriormente, se realizó el mismo abordaje al hombro izquierdo y reducción de la cabeza humeral, identificando y reparando de igual forma con dos anclas las lesiones del labrum y cápsula, pero en esta extremidad no se realizó osteosíntesis, porque no se consideró necesario, ya que se logró una adecuada impactación de la cabeza humeral a la diáfisis.

La paciente fue inmovilizada bilateralmente con cojines de abducción durante cuatro semanas y se inició un programa de rehabilitación progresiva por seis meses. Se realizaron controles radiográficos a las cuatro, ocho y 12 semanas, logrando recuperar el 90\% de los arcos de movimiento con mínima limitación para la extensión, pero sin que afectara sus actividades cotidianas. Fue valorada posteriormente a los seis y 12 meses postoperatorios y fue dada de alta definitiva (Figura 3). La paciente fue seguida por cinco años sin que refiriera limitación alguna.

\section{Caso 2}

Se presenta otro caso de un hombre de 54 años con antecedente de alcoholismo crónico, quien fue ingre- sado al hospital por cuadro de gastroenteritis aguda. Durante su internamiento presentó crisis convulsivas severa, que fue presenciada por el equipo médico y controlada después de más de 10 minutos.

En el periodo postictal, refirió dolor severo de articulación glenohumeral bilateral con limitación de movimientos y deformidad en hombros por luxación glenohumeral bilateral. Se le realizaron estudios de imagen que consistieron en radiología simple; se identificó la luxación posterior bilateral con fractura por avulsión de ambas tuberosidades mayores $(\mathrm{Fi}$ gura 4). Se complementó con estudio con tomografía axial y se comprobaron las lesiones. Se descartó evento vascular cerebral y, en coordinación con el equipo de Medicina Interna, se decidió realizar reducción abierta de ambos hombros ese mismo día.

Se realizó abordaje deltopectoral en tiempos secuenciados, reducción abierta y osteosíntesis con una placa bloqueada bilateral además de reparación de cápsula. En el hombro derecho se realizó tenotomía y reparación del tendón subescapular además de fijación de la tuberosidad mayor mediante suturas. En el hombro izquierdo el procedimiento fue similar, pero sin tenotomía (Figura 5).

Se mantuvo al paciente inmovilizado con cojines de abducción durante seis semanas y a las cuatro semanas inició un programa de rehabilitación con movimientos progresivos. Fue seguido por tres meses y presentó necrosis avascular de ambas cabezas humerales; fue tratado con reemplazo articular bilateral por otro cirujano.

\section{DISCUSIÓN}

La luxación bilateral posterior del hombro, descrita por primera vez en 1902 por Mynter, ${ }^{1,2}$ es extrema-
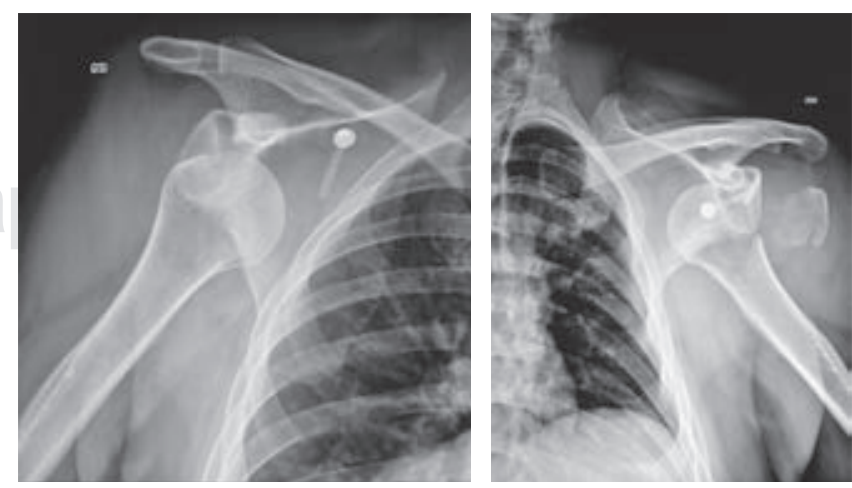

Figura 4: Radiografía anteroposterior de ambos hombros. Fractura luxación bilateral de húmero proximal con desplazamiento de ambas tuberosidades mayores. 


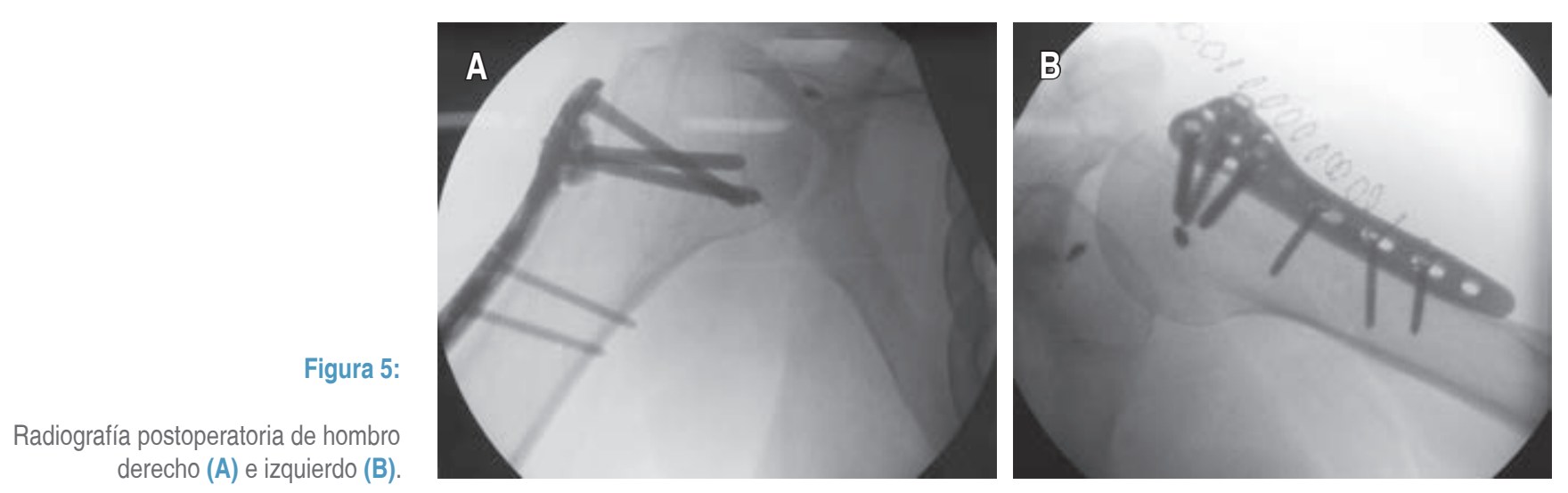

damente rara. La causa más común de esta situación son los eventos convulsivos. ${ }^{6}$ El mecanismo propuesto de lesión en el hombro durante una convulsión ha sido ya descrito. ${ }^{7}$

En una revisión de la literatura, que incluyó 8,140 casos de luxaciones de hombro, sólo se informaron 139 pacientes $(1.7 \%)$ con luxación posterior del hombro. ${ }^{5}$ Según Neer, ${ }^{4}$ ésta es la luxación más comúnmente omitida. Se distinguen tres tipos de luxación posterior: el tipo subacrominal como la más común, seguida por la luxación subglenoidea y la forma subespinosa.

La posición típica del hombro durante una convulsión es aducción, rotación interna y flexión. Con el espasmo, la cabeza humeral se desplaza proximalmente y posteriormente sobre la cavidad glenoidea. Si la convulsión se detiene, la cabeza humeral permanece bloqueada detrás de la cavidad glenoidea, que está asociada con la lesión de Hill Sachs invertida. La fuerza convulsiva adicional que actua en una cabeza humeral impactada contra el borde glenoideo puede resultar finalmente en una fractura proximal humeral compleja.

El diagnóstico de este tipo de lesión es comúnmente tardío y sólo $50 \%$ se identifica correctamente en la primera revisión, $, 8,9$ especialmente en un paciente con un primer episodio de convulsión, en estado postictal, el cual despierta con hombros rígidos y dolorosos, sin recordar ningún tipo de trauma. ${ }^{5}$ Una historia clínica detallada y un examen físico exhaustivo con al menos una vista de radiografía anteroposterior y axilar son esenciales al evaluar esta patología. ${ }^{2}$ En el momento del examen físico, el contorno del hombro puede ser normal con un proceso coracoideo prominente. El movimiento anormal doloroso de la articulación (con fractura o no) puede confundirse con el movimiento glenohumeral normal con rigidez postictal. Es importante dirigir la atención e identificar las posibles lesiones nerviosas asociadas.
La tomografía computarizada también facilita una imagen completa de la lesión y es de gran utilidad en la planificación y en el tratamiento, ${ }^{9}$ el cual debe ir dirigido al tipo de lesión, el intervalo de tiempo entre el trauma y el diagnóstico, la edad, la ocupación y los niveles de actividad y expectativas del paciente. ${ }^{5}$

Cuando la fractura se desplaza mínimamente y la viabilidad de la cabeza humeral tiene poco riesgo de necrosis según los criterios de Hertel, ${ }^{10-12}$ se debe realizar una reducción cerrada y, si es necesario, también la fijación del fragmento con clavillos de Kirschner. ${ }^{13,14}$ Se ha propuesto que tres semanas después del trauma la reducción cerrada es casi imposible y se requiere de manera forzosa una reducción quirúrgica. ${ }^{15}$

Para fracturas agudas desplazadas en pacientes jóvenes, si un intento de reducción cerrada no tiene éxito, la reducción abierta con fijación interna es la conducta a seguir. Si no se puede lograr una reducción abierta o en los casos en que más del $50 \%$ de la superficie articular de la cabeza está dañada, o si el paciente es mayor a 65 años con fracturas de tres o cuatro partes (lo que genera un alto riesgo de necrosis avascular) se debe considerar el reemplazo articular protésico como una de las opciones, dependiendo de la integridad muscular y el tipo de fractura o luxación; también debe considerarse si el reemplazo será total o parcial. ${ }^{5,6,16}$

Cuando el diagnóstico se retrasa durante varias semanas o meses y no existe evidencia de necrosis avascular, se pueden realizar otros procedimientos como: osteotomía humeral para corregir la mala unión y un procedimiento de McLaughlin o reconstrucción con aloinjerto del defecto de la cabeza humeral (lesión residual de Hill-Sachs reverso) para mantener una reducción estable. ${ }^{17-20}$ Algunos autores están de acuerdo, dependiendo de los hallazgos pre e intraoperatorios y del reemplazo articular del hombro cuando la duración de la luxación es mayor a seis meses. ${ }^{15,18,21-23}$ 


\section{CONCLUSIÓN}

La luxación posterior bilateral del hombro es una lesión poco común, además la limitación funcional de ambos hombros puede llegar a ser devastadora, más aún si éstas se asocian con una o más fracturas. Es esencial una sistematización constante del enfoque clínico y radiológico de los hombros lesionados ante la sospecha clínica de la luxación posterior para así evitar pasar por alto estas lesiones. El diagnóstico temprano y el tratamiento quirúrgico adecuado, realizado por un cirujano experimentado en tiempo y forma correcta contribuye a los resultados funcionales finales.

\section{BIBLIOGRAFÍA}

1. Dubousset J. Luxation posterièures de l'epaule. Rev Chir Orthop. 1967; 53: 65-85.

2. Brackstone M, Patterson SD, Kertesz A. Triple "E" syndrome: bilateral locked posterior fracture dislocation of the shoulders. Neurology. 2001; 56: 1403-1404.

3. Neer CS. Displaced proximal humeral fractures-Part II: treatment. J Bone Joint Surg Am. 1970; 52: 1090-1103.

4. Neer CS 2nd. Displaced proximal humeral fractures-Part I: classification and evaluation. J Bone Joint Surg Am. 1970; 52 : 1077-1089.

5. Cooke SJ, Hackney RG. Bilateral posterior four-part fractured dislocations of the shoulders following electric shock: a case report and literature review. Injury. 2005; 36: 90-95.

6. Djurdjevic D. Unrecognized posterior dislocation of the shoulder. Masters Thesis, Zagreb, 2003.

7. Hashmi FR, Pugh M, Bryan S. Simultaneous bilateral posterior dislocation of shoulder. Am J Emerg Med. 2002; 20: 127-128.

8. Shaw JL. Bilateral posterior fracture-dislocation of the shoulder and other trauma caused by convulsive seizures. J Bone Joint Surg Am. 1971; 53: 1437-1440.

9. Wadlington VR, Hendrix RW, Rogers LF. Computed tomography of posterior fracture-dislocations of the shoulder: case reports. J Trauma. 1992; 32: 113-115.
10. Duparc F, Muller JM, Fréger P. Arterial blood supply of the proximal humeral epiphysis. Surg Radiol Anat. 2001; 23: 185-190.

11. Gerber C, Schneeberger AG, Vinh TS. The arterial vascularisation of the humeral head: An anatomical study. J Bone Joint Surg Am. 1990; 72: 1486-1494.

12. Hertel R, Hempfing A, Stiehler M, Leunig M. Predictors of humeral head ischemia after intracapsular fracture of the proximal humerus. J Shoulder Elbow Surg. 2004; 13: 427-433.

13. Bell HM. Posterior fracture-dislocation of the shoulder: a method of closed reduction: a case report. J Bone Joint Surg Am. 1965; 47: 1521-1524.

14. De Wall M, Lervick G, Marsh JL. Posterior fracturedislocation of the proximal humerus: treatment by closed reduction and limited fixation: a report of four cases. J Orthop Trauma. 2005; 19: 48-51.

15. Walch G, Boileau P, Martin B, Dejour H. Unreduced posterior dislocations and fracture-dislocations of the shoulder: a review of 30 cases. Rev Chir Orthop. 1990; 76: 546-558.

16. Dervin GF, Brunet JA, Healy DC. A modification of the McLaughlin procedure as a salvage for missed locked posterior fracture-dislocation of the humeral head: a case report. J Bone Joint Surg Am. 2002; 84: 804-808.

17. McLaughlin HL. Posterior dislocation of the shoulder. J Bone Joint Surg Am. 1952; 34: 584-590.

18. Nicola FG, Ellman H, Eckardt J, Finerman G. Bilateral posterior fracture-dislocation of the shoulder treated with a modification of the McLaughlin procedure: a case report. J Bone Joint Surg Am. 1981; 63: 1175-1177.

19. Page AE, Meinhard BP, Schulz E, Toledano B. Bilateral posterior fracture-dislocation of the shoulders: management by bilateral shoulder hemiarthroplasties. J Orthop Trauma. 1995; 9: 526-529.

20. Gerber C, Lambert SM. Allograft reconstruction of segmental defects of the humeral head for the treatment of chronic locked posterior dislocation of the shoulder. J Bone Joint Surg Am. 1996; 78: 376-382.

21. Checchia SL, Santos PD, Miyazaki AN. Surgical treatment of acute and chronic posterior fracture-dislocation of the shoulder. J Shoulder Elbow Surg. 1998; 7: 53-65.

22. Hawkins RJ, Neer CS, Pianta RM, Mendoza FX. Locked posterior dislocation of the shoulder. J Bone Joint Surg Am. 1987; 69: 9-18.

23. Sperling JW, Pring M, Antuna SA, Cofield RH. Shoulder arthroplasty for locked posterior dislocation of the shoulder. $J$ Shoulder Elbow Surg. 2004; 13: 522-527. 